\title{
Pesquisa Nacional de Saúde 2019: histórico, métodos e perspectivas \\ doi: 10.1590/S1679-497420200000500004
}

\section{National Health Survey 2019: history, methods and perspectives}

\author{
Encuesta Nacional de Salud 2019: historia, métodos y perspectivas
}

\author{
Sheila Rizzato Stopa ${ }^{1}$ - (1) orcid.org/0000-0001-8847-665X \\ Célia Landmann Szwarcwald ${ }^{2}$ - (1) orcid.org/0000-0002-7798-2095 \\ Max Moura de Oliveira ${ }^{1}$ - (1) orcid.org/0000-0002-0804-5145 \\ Ellen de Cassia Dutra Pozzetti Gouvea ${ }^{1}$ - ๑ orcid.org/0000-0003-0611-3738 \\ Maria Lúcia França Pontes Vieira ${ }^{3}$ - ๑ orcid.org/0000-0002-5284-6214 \\ Marcos Paulo Soares de Freitas ${ }^{3}$ - 10 orcid.org/0000-0002-3293-7303 \\ Luciana Monteiro Vasconcelos Sardinha ${ }^{1}$ - (1) orcid.org/0000-0002-3679-3618 \\ Eduardo Marques Macário' 1 - (1) orcid.org/0000-0002-6383-0365
}

'Ministério da Saúde, Departamento de Análise em Saúde e Vigilância de Doenças não Transmissíveis, Brasília, DF, Brasil ${ }^{2}$ Fundação Instituto Oswaldo Cruz, Instituto de Comunicação e Informação Científica e Tecnológica em Saúde, Rio de Janeiro, RJ, Brasil ${ }^{3}$ Instituto Brasileiro de Geografia e Estatística, Diretoria de Pesquisas, Rio de Janeiro, RJ, Brasil

\section{Resumo}

Este artigo apresenta o histórico e a construção da Pesquisa Nacional de Saúde (PNS) 2019, inquérito de base domiciliar realizado em parceria com o Instituto Brasileiro de Geografia e Estatística. 0 objetivo da PNS 2019 foi dotar o país de informações sobre os determinantes, condicionantes e necessidades de saúde da população brasileira. A amostra prevista foi de 108.525 domicílios particulares, considerando-se uma taxa de não resposta de 20\%. Seu questionário continha três partes, orientadas para (i) o domicílio, (ii) todos os moradores do domicílio, com enfoque na coleta de informações socioeconômicas e de saúde, e (iii) o morador selecionado (idade $\geq 15$ anos), sendo investigados estilos de vida, doenças crônicas, violências, entre outros temas, e aferidas medidas antropométricas (subamostra). As informações da PNS 2019 servirão de base para a (re)formulação de políticas de saúde e subsídio a ações e programas existentes do Sistema Único de Saúde.

Palavras-chave: Inquéritos Epidemiológicos; Estudos Transversais; Metodologia; Tamanho da Amostra; Características da População; Brasil.

Endereço para correspondência:

Sheila Rizzato Stopa - SRTV 702, Via W5 Norte, Ed. PO700, 6 Andar, Brasília, DF, Brasil. CEP: 70723-040

E-mail: sheila.stopa@saude.gov.br 


\section{Introdução}

Os inquéritos de saúde são essenciais para avaliar os sistemas de saúde sob a perspectiva do usuário. São ferramentas importantes para compreender as necessidades reais da população - condições de vida e saúde -, imprescindíveis no planejamento, criação e/ou aperfeiçoamento de programas e políticas de saúde. ${ }^{1,2}$

Entre os principais objetivos dos inquéritos de saúde está o de prover informações de saúde que, em sua maioria, não são registradas nos sistemas de informações em saúde. A insuficiência desses sistemas para suprir necessidades da gestão pública enfatiza a importância de coletar dados primários, por meio de inquéritos de saúde de base populacional. ${ }^{1,3}$

\section{Preferencialmente realizados com periodicidade constante, os inquéritos possibilitam consubstanciar informações como base de referência populacional na definição da vigilância e monitoramento de morbidades e seus \\ fatores de risco e proteção.}

Preferencialmente realizados com periodicidade constante, os inquéritos possibilitam consubstanciar informações como base de referência populacional na definição da vigilância e monitoramento de morbidades e seus fatores de risco e proteção. Outra característica desses inquéritos é a possibilidade de analisar as informações coletadas sob a ótica das desigualdades em saúde, orientando políticas com o propósito de alcançar maior equidade no acesso à saúde. ${ }^{4,5}$

Em países de alta renda, os inquéritos de saúde são realizados desde a década de 1960 , enquanto, nos países de média e baixa renda, tal prática é mais recente. No Brasil, o primeiro inquérito no âmbito da saúde realizou-se em 1974, na cidade de Ribeirão Preto, SP, sobre morbidade referida e utilização de serviços de saúde. ${ }^{6}$ Aquela primeira iniciativa propiciou diversas discussões metodológicas e estimulou projetos subsequentes, como o Estudo Nacional de Despesa Familiar, realizado nos anos de 1974 e $1975 .{ }^{7.8}$ Subsequentemente, outros inquéritos foram conduzidos, como as Pesquisas de Orçamento Familiar (POF), pesquisas na área de alimentação e saúde reprodutiva, como a Pesquisa Nacional sobre Saúde e Nutrição em 1989, e a Pesquisa
Nacional sobre Demografia e Saúde, implementada em 1986 e reeditada em 1996 e 2006 (Figura 1) ${ }^{7,8}$

No âmbito da Saúde, propriamente, cabe citar o apoio do Ministério da Saúde no financiamento dos Suplementos Saúde da Pesquisa Nacional por Amostra de Domicílios (PNAD), nos anos de 1998, 2003 e 2008.,8 Tais suplementos possibilitaram comparações temporais, além do monitoramento de indicadores de acesso e utilização dos serviços de saúde, gastos com saúde e morbidades; 9 outras temáticas foram incorporadas, ao longo das edições dos suplementos, como fatores de risco e proteção para doenças crônicas não transmissíveis, acidentes de trânsito e violências, uso de medicamentos e cobertura da Estratégia Saúde da Família. ${ }^{9}$

Apesar de as iniciativas supracitadas terem contribuído, consideravelmente, para 0 crescimento da compreensão e monitoramento de indicadores de saúde, e dada a crescente apropriação da metodologia de inquéritos pela comunidade científica brasileira, havia a necessidade de ampliar a capacidade de captação de informações e análise de resultados. Diante disso, considerou-se a oportunidade de desenvolver um inquérito de saúde de âmbito nacional, de modo a atender a demandas e prioridades do Ministério da Saúde.

\section{Pesquisa Nacional de Saúde}

Em 2003, o Comitê Temático sobre Informações de Base Populacional recomendou a realização de uma pesquisa nacional de saúde de base populacional para avaliar condições de saúde, 0 acesso e utilização dos serviços e os gastos com saúde, a vigilância de doenças e agravos não transmissíveis e seus determinantes, além de questões sobre desigualdades sociais em saúde. Naquela ocasião, o Comitê Temático salientou a importância da previsão de recursos com periodicidade regular, para a sustentabilidade de uma pesquisa de tamanha abrangência e relevância. ${ }^{10}$

Posteriormente, em 2007, no seminário da Associação Brasileira de Saúde Coletiva (ABRASCO) sobre inquéritos de saúde, o processo de uma pesquisa nacional voltou a ser tema de destaque, quando foi recomendada a definição de um grupo de trabalho para seu planejamento e execução. No ano de 2009, o Ministério da Saúde promulgou a portaria que instituiu o Comitê Gestor para a estruturação de diretrizes na gestão da Pesquisa Nacional de Saúde (PNS) ${ }^{11}$ 
Em 2011, a portaria ministerial foi atualizada e incluiu as seguintes secretarias na condução da pesquisa: de Vigilância em Saúde (SVS), Executiva (SE), de Atenção à Saúde (SAS); de Gestão Estratégica e Participativa (SGEP), de Ciência, Tecnologia e Insumos Estratégicos (SCTIE); e, juntamente com elas, a Fundação Instituto Oswaldo Cruz (Fiocruz). ${ }^{12}$

Em 2013, foi a campo a primeira edição da PNS, com o propósito de ampliar a investigação temática dos Suplementos Saúde da PNAD. 0 questionário da PNS incluiu, integralmente, os módulos de acesso e utilização de serviços de saúde e cobertura de planos de saúde, principais investigações da PNAD, e possibilitou 0 acompanhamento da série quinquenal de seus indicadores no período 1998-2013. Ademais, de forma diferente à dos Suplementos Saúde, a PNS foi inserida no Sistema Integrado de Pesquisas Domiciliares (SIPD) do Instituto Brasileiro de Geografia e Estatística (IBGE), independentemente da amostra da PNAD Contínua, com desenho próprio e elaborada especialmente para coletar dados de saúde. Definiu-se a periodicidade de cinco anos para a realização de cada edição da PNS.

A coleta dos dados, mediante entrevistas, ocorreu entre agosto de 2013 e fevereiro de 2014. Conduzida pelo IBGE, ela envolveu mais de 1 mil técnicos da instituição, que coletaram dados em 1.600 municípios brasileiros. No total, quatro relatórios foram divulgados e, conjuntamente com as bases de dados, foram disponibilizados no sítio eletrônico do IBGE, vindo a ser empregados em diversas publicações técnico-científicas. ${ }^{13-16}$

Por ocasião do lançamento dos relatórios da PNS, em 2014 e 2015, o Ministério da Saúde logo manifestou interesse em conduzir uma segunda edição, de forma a permitir o monitoramento da evolução de seus indicadores de saúde sobre a situação investigada anteriormente, e a consequente resposta do Sistema Único de Saúde (SUS) no atendimento às necessidades da população.

Em abril de 2017, foi publicada a portaria ministerial que criou o Comitê Gestor da segunda edição da PNS, com a participação das seguintes secretarias: SVS, cabendo ao Departamento de Análise em Saúde e Vigilância de Doenças Não Transmissíveis a coordenação da pesquisa; SCTIE; SE; SAS; SGEP; de Gestão do Trabalho e da Educação na Saúde (SGTES); Especial de Saúde Indígena (SESAI); e a Fiocruz. ${ }^{17}$

O objetivo principal da PNS 2019 foi dotar o país de informações sobre os determinantes, condicionantes e necessidades de saúde da população brasileira, permitindo estabelecer medidas consistentes, capazes de auxiliar a elaboração de políticas públicas e alcançar maior efetividade nas intervenções em saúde. Os objetivos específicos da PNS 2019 estão descritos na Figura 2.

\section{Métodos}

A PNS 2019 é um inquérito de base populacional, representativo do Brasil e da população residente em domicílios particulares de seu território. Nela foi possível estimar os dados para as áreas urbana e rural, por grandes regiões nacionais, Unidades da Federação (UFs), capitais, e regiões metropolitanas. A PNS foi planejada para ter periodicidade quinquenal. Devido às questões relacionadas a seu delineamento e execução, a segunda edição, prevista inicialmente para 2018, só foi a campo no ano de 2019.

\section{Participantes}

A população-alvo da PNS consistiu dos indivíduos de 15 anos ou mais de idade, residentes em domicílios particulares permanentes, ou seja, construídos com a finalidade exclusiva de habitação. A amostra da pesquisa excluiu os domicílios localizados em setores censitários especiais ou de escassa população, como agrupamentos indígenas, quartéis, bases militares, alojamentos, acampamentos, embarcações, penitenciárias, colônias penais, presídios, cadeias, instituições de longa permanência para idosos, redes de atendimento integrado à criança e ao adolescente, conventos, hospitais, agrovilas de projetos de assentamento e agrupamentos quilombolas.

\section{Plano amostral e análise de dados}

A amostra da PNS originou-se de uma amostra mestra, constituída de um conjunto de unidades de áreas selecionadas em um cadastro, com 0 intuito de atender a seleções de subamostras para diversas pesquisas diferentes previstas no SIPD/IBGE, como a PNAD e a POF. Tais unidades são conceituadas como unidades primárias de amostragem (UPA), dentro do planejamento amostral das pesquisas que utilizam a amostra mestra, caso da PNS.

A estratégia de amostragem consistiu de (i) um plano conglomerado em três estágios, com estratificação das UPA (neste caso, setores censitários ou conjunto de setores) e seleção destas para a amostra mestra, 
com probabilidade proporcional ao tamanho, definida pelo número de domicílios particulares permanentes (DPP), e (ii) a seleção para a amostra da PNS, com probabilidade igualmente proporcional.

O segundo estágio consistiu da seleção de domicílios do Cadastro Nacional de Endereços para Fins Estatísticos (CNEFE), em sua atualização mais recente (realizada para a execução da PNAD Contínua 2019) antes da conclusão dessa etapa do plano amostral, por amostragem aleatória simples. A seguir, dentro de cada domicílio, um morador com 15 anos ou mais de idade foi selecionado aleatoriamente, com base na lista de moradores obtida no momento da entrevista. Para dimensionar o tamanho da amostra com o nível de precisão desejado para as estimavas, foram considerados alguns indicadores da edição de 2013 da PNS, como dados de DCNT (diabetes, hipertensão, depressão), violências, uso de serviços de saúde, posse de plano de saúde, tabagismo, prática de atividade física e consumo de álcool, entre outros. 0 tamanho final da amostra é apresentado na Tabela 1.

Para a definição do tamanho da amostra de UPA $\left(m_{d}\right)$, foi estabelecido o número de domicílios selecionados em cada UPA e, então, dividido o tamanho da amostra de domicílios por esse número: 12, 15 ou 18, a depender do domínio - subgrupos populacionais, para os quais são planejadas estimativas separadas:

$$
m_{d}=\frac{n_{\mathrm{ACd}}}{12}, m_{d}=\frac{n_{\mathrm{ACd}}}{15} \text { ou } m_{d}=\frac{n_{\mathrm{ACd}}}{18}
$$

Onde $\mathbf{n}_{\text {ACd }}$ corresponde ao tamanho de amostra sob amostragem conglomerada por domínios.

Essa diferença deveu-se à limitação no tamanho da amostra de UPA pela amostra mestra.

Em função do desenho amostral complexo e com probabilidades distintas de seleção, para analisar os dados da PNS, faz-se necessária a definição de fatores de expansão ou pesos amostrais, tanto para domicílios quanto para os moradores selecionados. 0 peso final aplicado é um produto do inverso das expressões de probabilidade de seleção de cada estágio do plano amostral, o que inclui correção de não respostas e ajustes dos totais populacionais.

Amostras originárias de pesquisas por conglomerados devem ser analisadas em programas de análise estatística que possuam pacotes destinados a dados complexos, ou seja, que possuam um algoritmo capaz de considerar os efeitos da estratificação e da conglomeração na estimação dos indicadores e suas medidas de precisão.
Dessa forma, no momento da análise, devem-se considerar três variáveis para que os dados possam ser representativos da população da qual se pretende obter estimativas. São elas: UPA, estrato e peso.

A divulgação da base de dados da PNS 2019 é de governabilidade do IBGE, sendo ela incluída no endereço eletrônico da instituição, na página referente à PNS. Conjuntamente, divulga-se um dicionário de variáveis que permite identificar as variáveis supracitadas. Além disso, pesos distintos são elaborados para a análise de todos os moradores do domicílio e do morador selecionado. 0 uso de cada um deles depende do conjunto de dados para o qual se pretende realizar a análise, e da seção do questionário à qual o dado pertence.

\section{Questionário}

Foram realizadas diversas reuniões de revisão do questionário da PNS 2019, com a presença de especialistas e representantes de áreas técnicas do Ministério da Saúde. Na sequência, deu-se início à etapa de discussões com o IBGE, no intuito de aprimorar o fluxo de questões e a semântica do questionário.

Alguns blocos de questões foram alterados na segunda edição, outros, incluídos. Opções de resposta com baixo percentual em 2013, ou seja, que apresentaram coeficiente de variação alto (acima de 30\%), a ponto de não alcançar a precisão desejada, foram excluídas do questionário da segunda edição, quando pertinente. De maneira geral, a maior parte do questionário manteve comparabilidade com a edição de 2013. Uma alteração importante foi feita na coleta de dados: anteriormente, na PNS 2013, ela era dirigida a indivíduos na idade de 18 anos ou mais; na edição de 2019, decidiu-se selecionar para a entrevista aqueles de 15 anos ou mais (com exceção de três blocos temáticos).

0 questionário definitivo foi dividido em três seções: a) Questionário domiciliar

Questões sobre informações do domicilio e visitas domiciliares realizadas por agentes de endemias e equipe de Saúde da Família. Esta parte do questionário foi respondida pelo morador(a) identificado(a) como responsável pelo domicílio.

b) Questionário para a todos os moradores do domicílio Foram coletadas informações gerais sobre todos os moradores do domicílio. Essas informações foram referidas por um morador de idade igual ou superior a 18 anos, que respondeu pelos demais 
Tabela 1 - Tamanho planejado da amostra para a Pesquisa Nacional de Saúde (PNS), segundo os domínios de interesse, Brasil, grandes regiões e Unidades da Federação, 2019

\begin{tabular}{|c|c|c|c|c|c|c|}
\hline \multirow[b]{2}{*}{$\begin{array}{l}\text { Grandes regiões } \\
\text { e Unidades da } \\
\text { Federação }\end{array}$} & \multirow[b]{2}{*}{$\begin{array}{l}\text { UPA }^{a} \text { na } \\
\text { amostra } \\
\text { mestra }\end{array}$} & \multicolumn{5}{|c|}{ Tamanho da amostra da PNS } \\
\hline & & $\begin{array}{c}\text { Número } \\
\text { planejado de } \\
\text { UPA }^{\mathrm{a}}\end{array}$ & $\begin{array}{l}\text { Número de UPA } \\
\text { após alocação }\end{array}$ & $\begin{array}{l}\text { Número planejado } \\
\text { de domicílios } \\
\text { selecionados }\end{array}$ & $\begin{array}{c}\text { Número de domicílios } \\
\text { selecionados após } \\
\text { alocação }\end{array}$ & $\begin{array}{l}\text { Número esperado de } \\
\text { domicílios com entrevista } \\
\text { realizada ( } 20 \% \text { de não } \\
\text { resposta) }\end{array}$ \\
\hline Brasil & 15.096 & 7.968 & 8.036 & 107.628 & 108.525 & 86.820 \\
\hline Norte & 1.896 & 1.362 & 1.370 & 21.636 & 21.762 & 17.410 \\
\hline Rondônia & 264 & 174 & 177 & 2.610 & 2.655 & 2.124 \\
\hline Acre & 276 & 193 & 193 & 2.895 & 2.895 & 2.316 \\
\hline Amazonas & 360 & 263 & 263 & 3.945 & 3.945 & 3.156 \\
\hline Roraima & 156 & 156 & 156 & 2.808 & 2.808 & 2.246 \\
\hline Pará & 504 & 330 & 333 & 4.950 & 4.995 & 3.996 \\
\hline Amapá & 108 & 108 & 108 & 1.944 & 1.944 & 1.555 \\
\hline Tocantins & 228 & 138 & 140 & 2.484 & 2.520 & 2.016 \\
\hline Nordeste & 4.908 & 2.810 & 2.840 & 37.104 & 37.503 & 30.002 \\
\hline Maranhão & 900 & 476 & 482 & 5.712 & 5.784 & 4.627 \\
\hline Piauí & 324 & 204 & 207 & 3.060 & 3.105 & 2.484 \\
\hline Ceará & 780 & 476 & 482 & 5.712 & 5.784 & 4.627 \\
\hline Rio Grande do Norte & 300 & 235 & 237 & 3.525 & 3.555 & 2.844 \\
\hline Paraíba & 384 & 240 & 245 & 3.600 & 3.675 & 2.940 \\
\hline Pernambuco & 600 & 374 & 380 & 4.488 & 4.560 & 3.648 \\
\hline Alagoas & 564 & 251 & 251 & 3.765 & 3.765 & 3.012 \\
\hline Sergipe & 288 & 198 & 201 & 2.970 & 3.015 & 2.412 \\
\hline Bahia & 768 & 356 & 355 & 4.272 & 4.260 & 3.408 \\
\hline Sudeste & 4.092 & 1.874 & 1.901 & 23.328 & 23.655 & 18.924 \\
\hline Minas Gerais & 1.104 & 488 & 495 & 5.856 & 5.940 & 4.752 \\
\hline Espírito Santo & 600 & 280 & 281 & 4.200 & 4.215 & 3.372 \\
\hline Rio de Janeiro & 1.164 & 508 & 512 & 6.096 & 6.144 & 4.915 \\
\hline São Paulo & 1.224 & 598 & 613 & 7.176 & 7.356 & 5.885 \\
\hline Sul & 2.664 & 1.090 & 1.090 & 13.080 & 13.080 & 10.464 \\
\hline Paraná & 828 & 373 & 374 & 4.476 & 4.488 & 3.590 \\
\hline Santa Catarina & 948 & 355 & 356 & 4.260 & 4.272 & 3.418 \\
\hline Rio Grande do Sul & 888 & 362 & 360 & 4.344 & 4.320 & 3.456 \\
\hline Centro-0este & 1.536 & 832 & 835 & 12.480 & 12.525 & 10.020 \\
\hline Mato Grosso do Sul & 336 & 222 & 225 & 3.330 & 3.375 & 2.700 \\
\hline Mato Grosso & 396 & 194 & 193 & 2.910 & 2.895 & 2.316 \\
\hline Goiás & 528 & 223 & 223 & 3.345 & 3.345 & 2.676 \\
\hline Distrito Federal & 276 & 193 & 194 & 2.895 & 2.910 & 2.328 \\
\hline
\end{tabular}

a) UPA: unidade primária de amostragem.

(proxy). Foram investigados: nível de escolaridade; informações sobre ocupaçãa; rendimentos domiciliares; deficiência física e/ou intelectual; cobertura de planos de saúde; acesso e utilização dos serviços de saúde; saúde dos indivíduos com 60 anos ou mais; e saúde de crianças com menos de 2 anos de idade.

c) Questionário do morador selecionado

Este bloco de questões destinou-se a um morador selecionado, com 15 ou mais anos de idade. Foram investigadas informações sobre características de trabalho e apoio social, percepção do estado de saúde, acidentes, estilos de vida, doenças crônicas não transmissíveis, saúde da mulher, atendimento pré-natal, saúde bucal, paternidade e pré-natal do parceiro, para pessoas com 15 anos ou mais; as questões sobre violências, doenças transmissíveis, atividade sexual, relações e condições de trabalho, e atendimento médico, foram respondidas apenas por aqueles com 18 anos ou mais. A Figura 3 elenca os módulos do questionário, contemplados nas edições de 2013 e 2019 da PNS.

Por fim, foi realizado pré-teste do instrumento de coleta da PNS 2019 com pequenas amostras de indivíduos, processo conduzido e desenvolvido pelos 


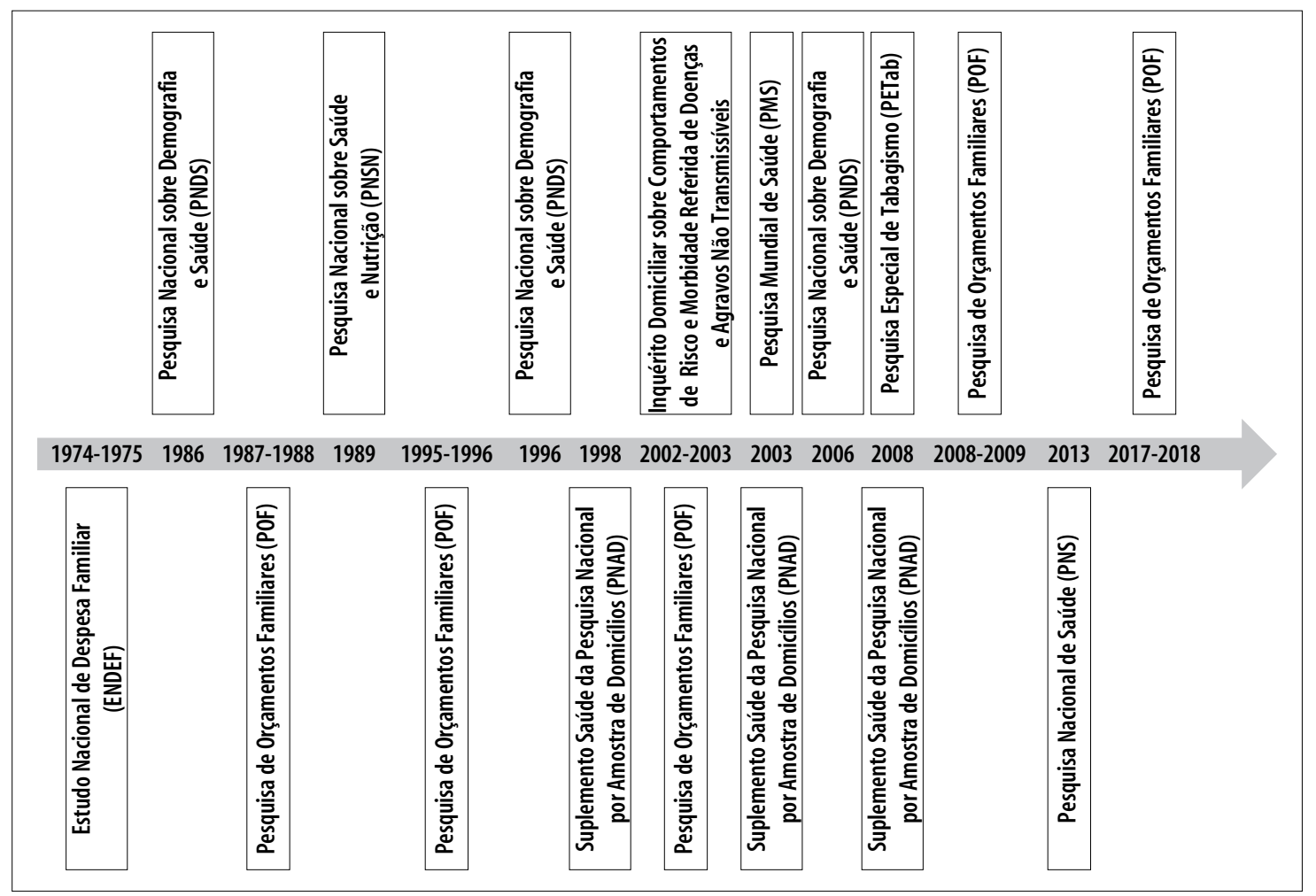

Figura 1 - Linha do tempo dos principais inquéritos domiciliares realizados no Brasil, 1974-2018

responsáveis da PNS nas unidades estaduais do IBGE. As novas questões introduzidas pela PNS 2019 ainda passaram por mais um processo de validação, 0 teste cognitivo, realizado em uma comunidade de baixo nível socioeconômico na cidade do Rio de Janeiro, RJ. Esse teste teve como objetivo avaliar a compreensão de cada questão a ser apresentada aos entrevistados, com a finalidade de obter a real percepção do instrumento e a necessidade de ajustes, caso necessários.

\section{Coleta de dados}

A organização das coletas e a coordenação do trabalho de campo, realizadas pelo IBGE, envolveram agentes de coleta (entrevistadores), supervisores (supervisão da coleta de dados e gestão dos agentes de coleta) e coordenadores (responsáveis pela pesquisa em determinada unidade estadual ou central) do próprio quadro de funcionários do IBGE.

0 treinamento dos coordenadores e demais envolvidos constituiu-se de etapas: em um primeiro momento, foram treinados os coordenadores das unidades estaduais do
IBGE por meio de oficina presencial realizada na cidade de Bento Gonçalves, RS. Os participantes tornaram-se agentes multiplicadores e, a retornarem a suas unidades, repassaram o conteúdo do treinamento aos supervisores e agentes de coleta. A equipe de campo (coordenadores, supervisores e agentes de coleta) participou, ao longo do processo da coleta de dados, de um treinamento simultâneo, com a possibilidade de questionamentos on-line.

As entrevistas foram realizadas com a utilização de dispositivos móveis de coleta (DMC), programados para "pulos" sobre itens do questionário e para análise crítica das variáveis. Ao chegar no domicílio selecionado, primeiramente 0 (a) agente de coleta fazia contato com o(a) responsável ou outro(a) morador(a). 0 (a) agente explicava aos moradores os objetivos, o procedimento de coleta de dados e a importância de sua participação na pesquisa. Nesse momento, era preenchida uma lista de todos os indivíduos residentes no domicílio, independentemente de concordarem ou não em participar da pesquisa.

Em seguida, identificava-se o(a) morador(a) que proveria as informações sobre os questionários 
a) Coletar informações sobre 0 acesso e 0 uso dos serviços de saúde, continuidade e longitudinalidade dos cuidados, com o objetivo de avaliar 0 desempenho dos sistemas de saúde.

b) Estimar a cobertura de planos de saúde, dimensionando a população segurada nas desagregações geográficas propostas para este estudo.

c) Delinear o perfil de necessidade de saúde das pessoas com 60 anos e mais, com base no autorrelato de restrições das atividades habituais.

d) Dimensionar a necessidade de cuidados para a realização das atividades habituais entre as pessoas com 60 anos ou mais de idade.

e) Dimensionar as prevalências de deficiências física e intelectual autorreferidas, além de funcionalidades.

f) Investigar os estilos de vida da população brasileira em relação a alimentação, tabagismo, consumo de bebidas alcoólicas e prática de atividade física.

g) Estabelecer os padrões de sobrepeso e obesidade da população brasileira com base na aferição de medidas.

h) Estimar a cobertura de exames preventivos de câncer de colo de útero e câncer de mama.

i) Dimensionar a exposição da população brasileira aos acidentes de trânsito, acidentes de trabalho e violências.

j) Estimar a prevalência das principais doenças crônicas não transmissíveis e as limitações provocadas por elas.

k) Investigar a atenção fornecida aos doentes diagnosticados com hipertensão, diabetes, colesterol alto e depressão, incluindo acesso a medicamentos, exames complementares de diagnóstico e continuidade nos cuidados.

I) Avaliar a atenção materno-infantil em relação ao atendimento pré-natal, assistência ao parto e cuidados preventivos e terapêuticos das crianças com menos de 2 anos de idade.

m) Identificar os determinantes que influenciam a estratificação social das condições de saúde, a exposição a fatores de risco à saúde, bem como a capacidade de resposta do sistema de saúde brasileiro.

n) Investigar aspectos relacionados à saúde em todo o ciclo de vida (saúde da criança, da mulher, do homem e do idoso).

0) Investigar 0 acesso à Atenção Primária à Saúde em termos de acesso, longitudinalidade, coordenação do cuidado e integralidade.

p) Estimar a prevalência de algumas doenças transmissíveis e de comportamentos relacionados à atividade sexual e ao uso de preservativos.

\section{Figura 2 - Objetivos específicos da Pesquisa Nacional de Saúde (PNS), Brasil, 2019}

\begin{tabular}{|l|c|c|}
\hline Módulos dos questionários & PNS 2013 & PNS2019 \\
\hline Módulo A Informaçóes do domicílio & $\mathrm{X}$ & $\mathrm{X}$ \\
\hline Módulo B Visitas domiciliares de equipe de Saúde da Família e agentes de endemias & $\mathrm{X}$ & $\mathrm{X}$ \\
\hline Módulo C Características gerais dos moradores & $\mathrm{X}$ & $\mathrm{X}$ \\
\hline Módulo D Características de educação dos moradores & $\mathrm{X}$ & $\mathrm{X}$ \\
\hline Módulo E Características de trabalho dos moradores & $\mathrm{X}$ & $\mathrm{X}$ \\
\hline Módulo F Rendimentos domiciliares & $\mathrm{X}$ & $\mathrm{X}$ \\
\hline Módulo G Pessoas com deficiências física e/ou intelectual & $\mathrm{X}$ & $\mathrm{X}$ \\
\hline Módulo I Cobertura de planos de saúde & $\mathrm{X}$ & $\mathrm{X}$ \\
\hline Módulo J Utilização de serviços de saúde & $\mathrm{X}$ & $\mathrm{X}$ \\
\hline Módulo K Saúde dos indivíduos com 60 anos ou mais de idade & $\mathrm{X}$ & $\mathrm{X}$ \\
\hline Módulo L Crianças com menos de 2anos de idade & $\mathrm{X}$ & $\mathrm{X}$ \\
\hline Módulo M Outras características do trabalho e apoio social & $\mathrm{X}$ & $\mathrm{X}$ \\
\hline Módulo N Percepção do estado de saúde & $\mathrm{X}$ & $\mathrm{X}$ \\
\hline Módulo O Acidentes & $\mathrm{X}$ & $\mathrm{X}$ \\
\hline Módulo P Estilos de vida & $\mathrm{X}$ & $\mathrm{X}$ \\
\hline Módulo Q Doenças crônicas & $\mathrm{X}$ & $\mathrm{X}$ \\
\hline Módulo R Saúde da mulher & $\mathrm{X}$ & $\mathrm{X}$ \\
\hline Módulo S Atendimento pré-natal & $\mathrm{X}$ & $\mathrm{X}$ \\
\hline Módulo U Saúde bucal & $\mathrm{X}$ & $\mathrm{X}$ \\
\hline Módulo Z Paternidade e pré-natal do parceiro & $\mathrm{X}$ \\
\hline Módulo V Violências & $\mathrm{X}$ & $\mathrm{X}$ \\
\hline Módulo T Doenças transmissíveis & $\mathrm{X}$ \\
\hline Módulo Y Atividade sexual & & \\
\hline Módulo AA Relações e condições de trabalho & \\
\hline Módulo X/H Atendimento médico & \\
\hline Módulo W Antropometria & & \\
\hline
\end{tabular}

a) Em 2013, 0 módulo ‘Atendimento médico' era 0 X, passando a ser definido como $H$ na edição de 2019 da pesquisa.

Figura 3 - Descrição dos módulos dos questionários da Pesquisa Nacional de Saúde (PNS), Brasil, 2013 e 2019 
domiciliar e de todos os moradores do domicílio, além do sorteio do(a) morador(a) de 15 anos e mais para responder à entrevista individual. As entrevistas foram agendadas segundo o horário mais conveniente para os moradores. Foram previstas duas ou mais visitas em cada domicílio.

A coleta de dados da PNS 2019 ocorreu entre os meses de agosto de 2019 e março de 2020.

\section{Aferições de medidas antropométricas}

Com relação à coleta de dados antropométricos, foram aferidos peso e altura apenas para a subamostra do morador selecionado, de 15 anos ou mais. Balanças, estadiômetros portáteis e gabaritos, além de fitas antropométricas, foram os materiais utilizados na coleta dessas medidas.

0 treinamento para a coleta de dados antropométricos foi desenvolvido em parceria com a Fiocruz e 0 Laboratório de Avaliação Nutricional de Populações (LANPOP) da Faculdade de Saúde Pública da Universidade de São Paulo (FSP/USP), e também foi ministrado durante $o$ treinamento de coordenadores e supervisores das unidades estaduais do IBGE.

Nesse treinamento, ressaltou-se a importância da calibração diária dos instrumentos, para a obtenção de medidas fidedignas. Toda a equipe de trabalho de campo do IBGE recebeu, além de treinamento presencial, material instrutivo (manuais e vídeos) sobre como proceder com a organização e calibração dos instrumentos, constituído de técnicas simples, como, por exemplo, a calibração da balança utilizando-se garrafas PET carregadas de água, de fácil verificação.

Para a coleta das medidas de peso e altura, os agentes de coleta foram instruídos sobre como proceder na abordagem dos moradores no domicílio, montagem dos equipamentos e obtenção das medidas. Para a aferição da altura, os agentes de coleta foram orientados a solicitar dos entrevistados que retirassem os sapatos e roupas pesadas e removessem adornos de cabelo. 0 (a) agente de coleta instruía a pessoa a permanecer ereta, pernas e pés paralelos e cabeça alinhada horizontalmente. Para a aferição do peso, os agentes foram orientados a solicitar aos entrevistados subirem na balança com os pés apoiados no centro da plataforma, mirando a linha do horizonte.

\section{Aspectos éticos}

A equipe de coleta de campo foi capacitada a compreender o que a pesquisa objetivava investigar, nas questões propostas e medidas aferidas. Agentes de coleta, bem como os coordenadores de campo, estavam aptos a responder às questões relativas a procedimentos previstos no estudo.

A PNS garantiu sigilo de identidade e dados pessoais dos participantes, moradores e entrevistados. Os consentimentos foram informados em duas etapas, ambas diretamente no DMC. Em um primeiro momento, para a entrevista domiciliar, o consentimento foi obtido antes da coleta das informações, pelo(a) informante do domićilio (proxy). Logo, um(a) morador(a) de 15 anos ou mais de idade foi selecionado(a) para a entrevista individual $\mathrm{e}$, caso concordasse em concedê-la, consentimentos eram obtidos para cada etapa: a entrevista propriamente, e a aferição das medidas antropométricas.

Cumpre lembrar que os selecionados entre $15 \mathrm{e}$ 17 anos não responderam às questões contidas nos módulos 'Violências', 'Doenças transmissíveis', 'Atividade sexual', 'Relações e condições de trabalho' e 'Atendimento médico'.

0 projeto da Pesquisa Nacional de Saúde 2019 foi encaminhado à Comissão Nacional de Ética em Pesquisa (CONEP)/Conselho Nacional de Saúde (CNS) e aprovado sob o Parecer $n^{0} 3.529 .376$, emitido em 23 de agosto de 2019.

\section{Considerações finais}

A PNS 2019 dá sequência às investigações no âmbito da saúde no país. É de extrema importância a continuidade do monitoramento de indicadores de saúde, e traduzi-los em políticas ou ações para 0 aprimoramento do SUS.

A primeira edição da PNS expandiu e aprofundou a investigação de questões de saúde, anteriormente trabalhadas em âmbito nacional, principalmente nos Suplementos Saúde da PNAD. Por ser uma pesquisa independente desde sua primeira edição, ou seja, desenhada com o intuito de coletar informações de saúde, a PNS estima os indicadores de interesse com a precisão desejada. Aqui, cabe ressaltar a comparabilidade entre os indicadores das duas edições, permitindo 0 
monitoramento de possíveis alterações nas estimativas, ao longo do período entre ambas as pesquisas.

Assim como em 2013, a PNS 2019 manteve 0 compromisso de dar sequência à investigação periódica das temáticas de acesso e utilização de serviços de saúde, além da cobertura de planos de saúde no país, iniciada em 1998 no Suplemento Saúde da PNAD. Dessa forma, são mais de 20 anos acompanhando os avanços e desafios nacionais na área. Por conseguinte, a PNS 2019 não apenas dá continuidade à investigação dessas temáticas; ela acompanha, no decorrer do tempo, os aspectos relativos às amostragens dos Suplementos Saúde. Assim, na medida em que se permite a comparação dos dados coletados entre as edições das duas pesquisas, é possível monitorar a evolução dos indicadores.

Nota-se a importância de aprofundar as questões de morbidade referida, mais do que estimar a prevalência na população, investigar a recomendação de medidas comportamentais que influenciam diretamente na saúde das pessoas, bem como 0 acesso a medicamentos e 0 uso regular dos serviços de saúde para acompanhamento de uma enfermidade. A possibilidade de monitorar tais dados, em diferentes períodos, permite conhecer a magnitude de uma doença ou agravo, o perfil de comportamentos adotados pela população acometida, primordiais no subsídio ao (re)planejamento de prioridades e ações em saúde. ${ }^{7,10}$ Nesse sentido, o Plano de Ações Estratégicas para o Enfrentamento das Doenças Crônicas Não Transmissíveis (DCNT) no Brasil 2011$2022,^{18}$ um dos principais instrumentos norteadores da vigilância de DCNT no país, incluiu ainda em 2011, momento de sua publicação, a realização de edições em 2013 e 2019 da PNS. 0 Plano de Ações Estratégicas reúne ferramentas qualificadas de aporte ao monitoramento das DCNT.

Um diferencial da PNS 2019 foi a alteração da faixa etária do morador selecionado para a entrevista. Esta alteração foi motivada pela necessidade de coletar dados válidos para monitoramento dos indicadores estabelecidos nos Objetivos de Desenvolvimento Sustentável (ODS). ${ }^{19}$ Não obstante, os indicadores a serem divulgados em publicações oficiais do IBGE e do Ministério da Saúde serão referentes à população de 18 anos ou mais, com 0 intuito de permitir comparação entre as edições. Indicadores pertinentes aos ODS também serão apresentados para a população de 15 anos ou mais.

Entre os novos módulos incorporados à PNS 2019, destacaram-se (i) as 'Doenças transmissíveis', com referência à presença de tosse persistente, manchas com dormência, doença de Chagas e infecção sexualmente transmissível, (ii) a 'Atividade sexual', que investigou iniciação sexual, uso e acesso a preservativos, (iii) o 'Atendimento médico', com enfoque no acesso e qualidade da Atenção Primária à Saúde (APS), e (iv) a 'Paternidade e pré-natal do parceiro', que abordou temáticas do acompanhamento pré-natal do parceiro, ${ }^{20}$ com 0 objetivo de ser uma porta de entrada dos homens na atenção básica à saúde. Todas essas temáticas foram demandadas por áreas técnicas do Ministério da Saúde.

0 módulo sobre 'Violências' também foi reformulado na PNS 2019, passando a investigar, separadamente, as violências psicológica, física e sexual, bem como a necessidade de uso de serviços de saúde em virtude de violência considerada mais grave. A temática da violência é considerada complexa e sensível, ${ }^{21} \mathrm{e}$, para garantir a privacidade da pessoa entrevistada, optou-se por solicitar que 0 (a) entrevistado(a) respondesse ao questionário diretamente no DMC. Caso a pessoa entrevistada não se sentisse confortável ou, ainda, tivesse alguma dificuldade em ler as questões diretamente no DMC, o(a) entrevistador(a) apresentaria os questionamentos. Nessas situações, $o$ (a) entrevistador(a) informava no DMC que a privacidade da entrevista não havia sido garantida, havendo a possibilidade de resposta na frente de terceiros. Além do módulo 'Violências', outros dois também foram respondidos pela própria pessoa entrevistada: 'Doenças transmissíveis' e 'Atividade sexual'.

Na PNS 2019, foram coletadas informações de peso e altura em uma subamostra. A despeito do número de medidas antropométricas reduzido, em relação à edição de 2013, os dados aferidos na PNS 2019 permitirão o monitoramento do excesso de peso e obesidade no Brasil (urbano e rural) e nas grandes regiões do país. Outros inquéritos, como o Sistema de Vigilância de Fatores de Risco e Proteção para Doenças Crônicas por Inquérito Telefônico ${ }^{22}$ (Vigitel), também investigam os mesmos dados, embora referidos pelo próprio(a) entrevistado(a). As estimativas da PNS, aferidas com equipamentos antropométricos calibrados, são consideradas padrão ouro. Assim, a realização de tais medidas torna-se essencial em grandes inquéritos domiciliares.

A PNS é um dos principais instrumentos utilizados pelo Ministério da Saúde para resposta/monitoramento de indicadores globais, incluindo os dos ODS, ${ }^{19}$ do Plano de Ações Globais para Prevenção e Controle das DCNT 
2013-202023 e do Plano de Ações Estratégicas para o Enfrentamento das Doenças Crônicas Não Transmissíveis (DCNT) no Brasil 2011-2022. ${ }^{18}$ Outrossim, a PNS é o padrão ouro dos inquéritos de saúde brasileiros, por ser a maior, mais completa e abrangente pesquisa de saúde no país. Outras pesquisas, a exemplo do mesmo Vigitel, ${ }^{22}$ fazem uso das estimativas da PNS para validarem os achados de seus monitoramentos. Assim, a edição de 2019 da PNS também permitirá nova avaliação da qualidade do Vigitel, principal inquérito de saúde anual realizado pelo Ministério da Saúde, utilizado no monitoramento dos fatores de risco à saúde da população brasileira.

Como limitações da PNS, além das clássicas, referentes aos estudos de desenho transversal - não determinação da causalidade, ou exclusão de população em situação de rua e asilada, p. ex. -, a PNS não estima dados para abrangências geográficas menores que as dos municípios das capitais, além do que, parte das temáticas investigadas na PNS (questionários do domicílio e de todos os moradores) são coletadas em entrevista com um(a) morador(a) do domicílio, que responde por si e pelos demais (proxy). Em relação ao morador selecionado, tão somente no caso de não ter condições de responder por motivos de saúde, física ou mental, foi solicitado a outro(a) morador(a) responder em seu lugar. Entretanto, estudos realizados com outros inquéritos de saúde demonstraram boa concordância e reprodutibilidade entre as informações coletadas por um proxy e as disponibilizadas pelo próprio indivíduo. ${ }^{24,25}$

\section{Referências}

1. Cesar CLG, Goldbaum M. Usos de serviços de saúde. In: Cesar CLG, Carandina L, Alves MCGP, Azevedo MB, Goldbaum M. Saúde e condição de vida em São Paulo: inquérito multicêntrico de saúde no Estado de São Paulo: ISA-SP. São Paulo: Faculdade de Saúde Pública da USP; 2005. p. 185-98.

2. Travassos CMR, Viacava F, Laguardia J. Os suplementos saúde na pesquisa nacional por amostra de domicílios (PNAD) no Brasil. Rev Bras Epidemiol [Internet]. 2008 [citado 2020 ago 17];11 Supl 1:98-112. Disponível em: https://doi.org/10.1590/\$1415790X2008000500010

3. Szwarcwald CL, Malta DC, Pereira CA, Vieira MLFP, Conde WL et al. Pesquisa Nacional de Saúde no
Planeja-se divulgar os resultados da PNS 2019 em relatórios oficiais do IBGE - em parceria com 0 Ministério da Saúde -, acompanhados de análises descritivas de indicadores previamente selecionados; e por intermédio da produção e publicação de material científico. Os resultados da PNS 2019 também deverão estar disponíveis em plataforma/painel de dados, com a possibilidade de tabulações simples, e nos bancos de dados no sítio eletrônico do IBGE, para análises mais complexas.

Realizar um inquérito de saúde da amplitude da PNS 2019 significa um avanço para a Saúde Pública brasileira. As informações obtidas desse trabalho servirão de subsídio para ações e políticas futuras do SUS, além do monitoramento e avaliação dos programas e serviços de saúde existentes.

\section{Contribuição dos autores}

Stopa SR contribuiu na concepção e delineamento do artigo e redigiu sua primeira versão. Szwarcwald CL, Oliveira MM, Gouvea ECDP e Freitas MPS contribuíram na escrita e revisão crítica do manuscrito. Vieira MLFP, Sardinha LMV e Macário EM revisaram criticamente 0 manuscrito. 0 Grupo Técnico da PNS 2019 apoiou 0 desenvolvimento da pesquisa em suas etapas e revisou a versão final do manuscrito. Todos os autores aprovaram a versão final e declaram ser responsáveis por todos os aspectos do trabalho, incluindo a garantia de sua precisão e integridade.

Brasil: concepção e metodologia da aplicação. Ciênc Saúde Coletiva [Internet]. 2014 fev [citado 2020 ago 14];19(2):333-42. Disponível em: https://doi. org/10.1590/1413-81232014192.14072012

4. Barreto ML. Desigualdades em saúde: uma perspectiva global. Ciênc Saúde Coletiva [Internet]. 2017 jul [citado 2020 jago 14];22(7):2097-108. Disponível em: https://doi.org/10.1590/141381232017227.02742017

5. Szwarcwald CL, Souza-Júnior PBR, Damacena GN. Socioeconomic inequalities in the use of outpatient services in Brazil according to health care need: evidence from the World Health Survey. BMC Health Serv Res [Internet]. 2010 [cited 2020 Aug 14];10:217. Available from: https://doi.org/10.1186/1472-6963-10-217 
6. Carvalheiro JR. Investigação epidemiológica e entrevistas domiciliarias. Rev Saúde Pública [Internet]. 1981 out [citado 2020 ago 14];15(5):54350. Disponível em: https://doi.org/10.1590/S003489101981000500007

7. Barros MBA. Inquéritos domiciliares de saúde: potencialidades e desafios. Rev Bras Epidemiol [Internet] 2008 maio [citado 2020 ago 14];11(supl1):6-19. Disponível em: https://doi. org/10.1590/S1415-790X2008000500002

8. Viacava F, Dachs N, Travassos C. Os inquéritos domiciliares e o sistema Nacional de Informações em Saúde. Ciênc Saúde Coletiva [Internet]. $2006 \mathrm{dez}$ [citado 2020 ago 14];11(4):863-9. Disponível em: https://doi.org/10.1590/\$1413-81232006000400002

9. Viacava F. Dez anos de informação sobre acesso e uso de serviços de sáude. Cad Saude Pública [Internet]. 2010 dez [citado 2020 ago 14];26(12):2210-1. Disponível em: https://doi.org/10.1590/S0102311X2010001200001

10. Malta DC, Leal MC, Costa MFL, Morais-Neto OL. Inquéritos nacionais de saúde: experiência acumulada e proposta para o inquérito de saúde brasileiro. Rev Bras Epidemiol [Internet]. 2008 maio [citado 2020 ago 14];11(suppl 1):159-67. Disponível em: https:// doi.org/10.1590/S1415-790X2008000500017

11. Brasil. Ministério da Saúde. Portaria MS/GM nº 1.811, de 12 de agosto de 2009. Institui, no âmbito do Ministério da Saúde, Comitê Gestor com a finalidade de planejar e coordenar a criação do Inquérito Nacional de Saúde - INS [Internet]. Diário Oficial da União, Brasília (DF), 2009 ago 12 [citado 2020 mar 27]. Disponível em: https://bvsms.saude.gov.br/bvs/ saudelegis/gm/2009/prt1811_12_08_2009.html

12. Brasil. Ministério da Saúde. Portaria MS/GMn ${ }^{0} 2.350$, de 5 de outubro de 2011. Institui, no âmbito no Ministério da Saúde, o Comitê Gestor da Pesquisa Nacional de Saúde (PNS) [Internet]. Diário Oficial da União, Brasília (DF), 2011 out 6 [citado 2020 abr 12];Seção 1:58 Disponível em: https://bvsms.saude.gov.br/bvs/ saudelegis/gm/2011/prt2350_05_10_2011.html

13. Instituto Brasileiro de Geografia e Estatística. Pesquisa Nacional de Saúde 2013: percepção do estado de saúde, estilos de vida e doenças crônicas [Internet]. Rio de Janeiro: Instituto Brasileiro de Geografia e Estatística; 2014 [citado 2020 fev 22]. 180 p. Disponível em: https:/biblioteca.ibge.gov.br/ visualizacao/livros/liv91110.pdf
14. Instituto Brasileiro de Geografia e Estatística IBGE. Pesquisa Nacional de Saúde 2013: acesso e utilização dos serviços de saúde, acidentes e violências [Internet]. Rio de Janeiro: Instituto Brasileiro de Geografia e Estatística; 2015 [citado 2020 fev 22]. 98 p. Disponível em: https://biblioteca.ibge.gov.br/ visualizacao/livros/liv94074.pdf

15. Instituto Brasileiro de Geografia e Estatística IBGE. Pesquisa Nacional de Saúde 2013: ciclos de vida [Internet]. Rio de Janeiro: Instituto Brasileiro de Geografia e Estatística; 2015 [citado 2020 fev 22]. 85 p. Disponível em: https://biblioteca.ibge.gov.br/ visualizacao/livros/liv94522.pdf

16. Instituto Brasileiro de Geografia e Estatística IBGE. Pesquisa Nacional de Saúde 2013: indicadores de saúde e mercado de trabalho [Internet]. Rio de Janeiro: Instituto Brasileiro de Geografia e Estatística; 2016 [citado 2020 fev 22] 66 p. Disponível em: https://biblioteca.ibge.gov.br/visualizacao/livros/ liv97329.pdf

17. Brasil. Ministério da Saúde. Portaria MS/GM n ${ }^{0}$ 1.002, de 13 de abril de 2017. Institui, no âmbito no Ministério da Saúde, o Comitê Gestor da Pesquisa Nacional de Saúde 2018 - PNS/2018 [Internet]. Diário Oficial da União, Brasília (DF), 2017 abr 13 [citado 2020 abr 09];Seção 1:30. Disponível em: https://bvsms.saude.gov.br/bvs/saudelegis/gm/2017/ prt1002_17_04_2017.html

18. Ministério da Saúde (BR). Secretaria de Vigilância em Saúde. Departamento de Vigilância de Doenças e Agravos Não Transmissíveis e Promoção da Saúde. Plano de ações estratégicas para o enfrentamento das doenças crônicas não transmissíveis (DCNT) no Brasil 2011-2022 [Internet]. Brasília: Ministério da Saúde; 2011 [citado 2020 ago 14]. 160 p. Disponível em: https://bvsms.saude.gov.br/bvs/publicacoes/ plano_acoes_enfrent_dent_2011.pdf

19. Organização das Nações Unidas - ONU. Objetivos de desenvolvimento sustentável [Internet]. [S.1]: Organização das Nações Unidas; 2017 [citado 2020 abr 13]. Disponível em: https://nacoesunidas.org/ pos2015/agenda2030/

20. Ministério da Saúde (BR). Secretaria de Atenção à Saúde. Departamento de Ações Programáticas Estratégicas. Coordenação Nacional de Saúde do Homem. Guia do pré-natal do parceiro para profissionais de saúde [Internet]. Brasília: Ministério da Saúde; 2016 [citado 2020 ago 14]. 55 p. Disponível em: https://portalarquivos2.saude.gov.br/images/ pdf/2016/agosto/11/guia_PreNatal.pdf 
21. Schraiber LB, d'Oliveira AFPL, Couto MT. Violência e saúde: contribuições teóricas, metodológicas e éticas de estudas da violência contra a mulher. Cad Saúde Pública [Internet]. 2009 [citado 2020 ago 14];25 Sup 2:S205-16. Disponível em: https://doi.org/10.1590/ S0102-311X2009001400003

22. Ministério da Saúde (BR). Secretaria de Vigilância em Saúde. Departamento de Análise em Saúde e Vigilância de Doenças Não Transmissíveis. Vigitel Brasil 2019: vigilância de fatores de risco e proteção para doenças crônicas por inquérito telefônico [Internet]. Brasília: Ministério da Saúde; 2020 [citado 2020 ago 14]. 137 p. Disponível em: http://www.crn1.org.br/wp-content/ uploads/2020/04/vigitel-brasil-2019-vigilancia-fatoresrisco.pdf? $\times 53725$

23. Organização Mundial da Saúde - OMS. Plano de ações globais para prevenção e controle das DCNT 2013-2020 [Internet]. Genebra: Organização

\section{Abstract}

This article presents the bistory and construction of the National Health Survey (PNS) 2019, a bousehold survey conducted in partnership with the Brazilian Institute of Geography and Statistics. The objective of PNS 2019 was to provide the country with information on the bealth determinants, conditionants and needs of the Brazilian population. The expected sample was 108,525 households, considering a 20\% non-response rate. The questionnaire had three parts, covering: (i) the bousehold; (ii) all residents of the bousebold, focusing on collection of socioeconomic and bealth information; and (iii) the selected resident (15 years old or more) for whom lifestyles, chronic diseases, violence, among other topics were investigated, as well as their anthropometric measurements (subsample). The information provided by PNS 2019 will serve as a basis for the (re)formulation of health policies, as well as support for existing actions and programs of the Brazilian National Health System.

Keywords: Health Surveys; Cross-Sectional Studies; Methodology; Sample Size; Population Characteristics; Brazil.
Mundial da Saúde; 2013 [citado 2020 ago 14].

Disponível em: https://apps.who.int/iris/bitstream/ handle/10665/94384/9789241506236 eng.pdf;jsessi onid $=$ A5E572CA1D2F8DE7F416EAAFDF6065D1?seq uence $=1$

24. Jardim R, Barreto SM, Giatti L. Confiabilidade das informações obtidas de informante secundário em inquéritos de saúde. Cad Saúde Pública [Internet]. 2010 [citado 2020 ago 14];26(8):1537-48. Disponível em: https://doi.org/10.1590/S0102311X2010000800008

25. Santana VS, Almeida Filho N, Rocha CO, Matos AS. Confiabilidade e viés do informante secundário na pesquisa epidemiológica: análise de questionário para triagem de transtornos mentais. Rev Saúde Pública [Internet]. $1997 \mathrm{dez}$ [citado 2020 ago 14];31(6):55665. Disponível em: https://doi.org/10.1590/S003489101997000700003

\section{Resumen}

Este artículo presenta la bistoria y la construcción de la Encuesta Nacional de Salud (PNS) 2019, una encuesta domiciliaria realizada en colaboración con el Instituto Brasileño de Geografía y Estadística. El objetivo de la PNS fue proporcionar al país información sobre los determinantes, las condiciones y las necesidades de salud de la población brasileña. La muestra esperada fue de 108.525 bogares particulares, considerando una tasa de no respuesta del 20\%. Su cuestionario contenía tres partes: (i) con respecto al hogar; (ii) dirigido a todos los residentes, centrándose en la recopilación de información socioeconómica y de salud;y (iii) al residente seleccionado (15 años o más) de quien se investigó estilos de vida, enfermedades crónicas, violencia, entre otros temas y se compararon las medidas antropométricas (submuestra). La información de la PNS 2019 servirá como base para la (re)formulación de políticas de salud y para apoyar acciones y programas existentes en el Sistema Único de Salud.

Palabras clave: Encuestas Epidemiológicas; Estudios Transversales; Metodología; Tamaño de la Muestra; Características de la Población; Brasil.

Recebido em 18/05/2020

Aprovado em 09/07/2020 